\title{
Primary Small Intestinal Natural Killer/T Cell Lymphoma Mimicking Tuberculous Peritonitis: Report of a Case and Review of the Literature
}

\author{
Yen-Nien Lin ${ }^{1}$, Jen-Wei Chou ${ }^{2}$, Po-Heng Chuang ${ }^{2}$, Ken-Sheng Cheng ${ }^{2}$, \\ Cheng-Yuan Peng ${ }^{2}$ and I-Ping Chiang ${ }^{3}$
}

\begin{abstract}
Extranodal natural killer/T cell lymphoma is very rarely encountered in clinical practice. It has a high mortality rate and very short median survival. Early diagnosis of these rare tumors, especially those originating from the small intestine, is usually difficult because of its nonspecific symptoms. Herein, we describe a case of a primary small intestinal natural killer/T cell lymphoma in a 52-year-old man who presented with abdominal fullness and weight loss. The clinical symptoms, elevation of serum levels of cancer antigen-125, and presence of ascites initially led to the suspicion of tuberculous peritonitis. Abdominal computed tomography scan demonstrated a hypodense tumor in the jejunum. Finally, the tumor was surgically confirmed to be a natural killer/T-cell lymphoma. Although aggressive chemotherapy was prescribed, the patient subsequently died of disease progression. In addition, we also review the English literature on this rare disease.
\end{abstract}

Key words: small intestinal natural killer/T cell lymphoma, non-Hodgkin's lymphoma, cancer antigen-125, tuberculous peritonitis, adenosine deaminase assay

(Intern Med 50: 515-518, 2011)

(DOI: 10.2169/internalmedicine.50.4435)

\section{Introduction}

Gastrointestinal (GI) lymphomas are the most common type of primary extranodal lymphomas, accounting for onefifth of all non-Hodgkin's lymphomas (NHL). They are almost always of the B-cell lineage. Natural killer/T-cell lymphomas (NK/T-Ls), especially those occurring in the GI tract, are rare; recently some cases have been described in a few reports $(1,2)$. Tuberculous (TB) peritonitis is a subacute disease and its symptoms evolve over several weeks to a few months. The clinical features of TB peritonitis are nonspecific and include abdominal pain, fever, weight loss, and diarrhea. Elevated serum levels of cancer antigen (CA)-125 has been documented in this disease and was subsequently recommended as an indirect marker for disease progression (4-6). Herein, we describe a rare case of primary small intestinal NK/T-L that mimicked TB peritonitis. We also emphasize the diagnostic value of CA-125 and early surgical intervention in patients with extranodal NK/T-L.

\section{Case Report}

A 52-year-old man was admitted to our hospital for a 2month history of abdominal fullness, fever, and weight loss. He had a medical history of hyperlipidemia and hypertension, but denied history of alcohol consumption or drug abuse. Both his grandfather and uncle had a history of pulmonary TB. Two months prior to admission, he developed symptoms of easy satiety, poor appetite, and abdominal fullness. He visited another hospital where esophagogastroduodenoscopy (EGD) and colonoscopy showed no remarkable abnormalities. Laboratory tests showed the presence of occult blood in his stools. Thereafter, he developed dry cough,

\footnotetext{
${ }^{1}$ Department of Internal Medicine, China Medical University Hospital, China Medical University, Taiwan, ${ }^{2}$ Division of Gastroenterology and Hepatology, Department of Internal Medicine, China Medical University Hospital, China Medical University, Taiwan and ${ }^{3}$ Department of Pathology, China Medical University Hospital, China Medical University, Taiwan

Received for publication August 28, 2010; Accepted for publication November 1, 2010

Correspondence to Dr. Jen-Wei Chou, codecol@yahoo.com.tw
} 


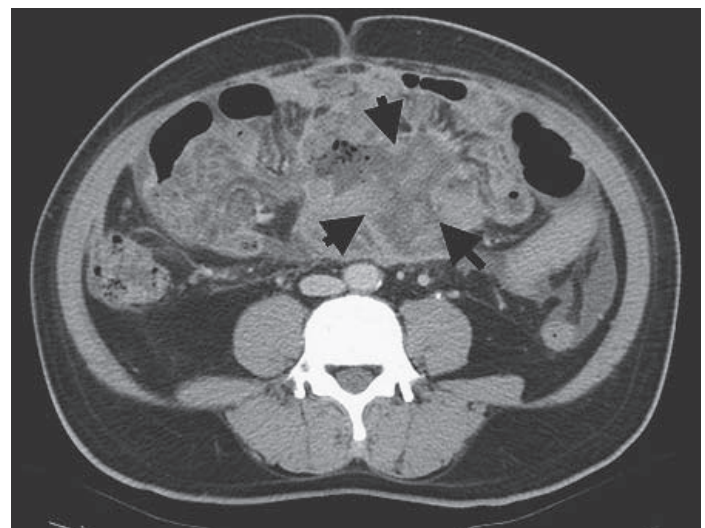

Figure 1. Abdominal computed tomography scan showed a cavitary mass with thickening of the bowel wall in the lower abdominal wall (arrows) and omental cake appearance.

malaise, and dyspnea on exertion. Low-grade fever, night sweats, and loss of weight by $14 \mathrm{~kg}$ were also noted during this period. On examination, his chest showed bilaterally decreased breath sounds at the lung bases. His abdomen was hard and tender without organomegaly. Shifting dullness and rebound tenderness were also noted. Enlargement of the cervical or axillary lymph nodes were identified. Laboratory tests revealed microcytic anemia ( $\mathrm{Hb}: 12.6 \mathrm{~g} / \mathrm{dL}$, normal 14$18 \mathrm{~g} / \mathrm{dL}$ ), and hypoalbuminemia (albumun: $2.4 \mathrm{~g} / \mathrm{dL}$, normal 3.4-4.8 g/dL). The white blood cell count, platelet count, results of coagulation studies, liver and renal function tests, and levels of electrolytes were normal. The fecal occult blood test by using enzyme immunoassay was $214.6 \mathrm{ng} / \mathrm{mL}$ (normal, $<12 \mathrm{ng} / \mathrm{mL}$ ). A chest radiograph and thoracic ultrasonography revealed bilateral moderate pleural effusions. Abdominal ultrasonography demonstrated thickened peritoneum with a moderate amount of ascites without cirrhosis of the liver. Diagnostic paracentesis and thoracocentesis both yielded exudative fluid that predominantly contained mononuclear cells. The serum level of CA-125 was $727.2 \mathrm{U} / \mathrm{mL}$ (normal, $<35 \mathrm{U} / \mathrm{mL}$ ), while the serum carcinoembryonic antigen (CEA) and CA-199 levels were within the normal range. The level of CA-125 in the ascitic fluid was markedly elevated at $2,008.5 \mathrm{U} / \mathrm{mL}$. Although the results of acidfast staining tests were negative in the ascites and pleural effusions, TB peritonitis was initally suspected on the basis of the patient's symptoms, and elevated CA-125 level in the serum and ascites. However, the adenosine deaminase (ADA) level in the ascitic fluid was $30 \mathrm{U} / \mathrm{mL}$. In addition, TB-PCR and TB culture tests of ascitic fluid also yielded a negative finding. The analysis of the ascitic fluid did not reveal growth of common microorganisms or the presence of malignant cells. Abdominal computed tomography (CT) scan revealed a cavitary mass with thickening of the bowel wall and air fluid level in the lower abdomen, ascites with omental cake appearance, as well as multiple enlarged mesenteric lymph nodes (Fig. 1). A barium study of the small intestine showed a cavitary mass lesion with mucosal destruction between the distal jejunum and proximal ileum,

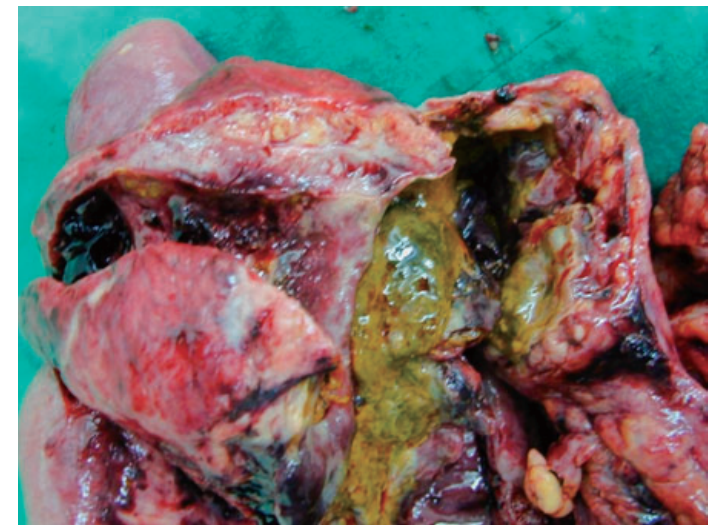

Figure 2. A mass which originated from the jejunum was seen with thickening of the segmental wall, mucosal disruption, and intestinal perforation with omental wrapping.

and focal segmental jejunal wall rigidity with lost of mucosal folds. He underwent a laparotomy, which revealed the following: a mass arising from the jejunum $90 \mathrm{~cm}$ distal to Treitz ligament, thickening of the segmental wall to $10 \mathrm{~cm}$, mucosal disruption and perforation with omental wrapping (Fig. 2). The surrounding portion of the mesentery was thickened and fragile. There were multiple enlarged lymph nodes. Invasion of the mesentery of the cecum was also noted. Resection of the small bowel and partial colectomy were performed. Microscopically, the resected specimens revealed a monotonous population of lymphoid cells with scanty cytoplasm. Necrosis and hemorrhage were noted. The immunohistochemical study was positive for leukocyte common antigen (LCA), CD3 (Fig. 3A), and CD56 (Fig. 3B), but negative for $\mathrm{CD} 20$. On the basis of the pathological characteristics, a diagnosis of NK/T-L was made. A FDG positron emission tomography (PET) whole body scan showed intensely increased FDG uptake involving most of the intra-abdominal region (omentum/mesentary/peritoneum) and in the lumbar pre/para-vertebral region (retroperitoneal lymph nodes). The patient developed short bowel syndrome after surgery. He received several courses of adjuvant chemotherapy, but still died of disease progression 9 months later.

\section{Discussion}

According to the World Health Organization classification of lymphoid malignancies, NK/T-L is classified as a mature T-cell and NK-cell neoplasm. Extranodal NK/T-L is a rare entity, which generally pursues an aggressive course, and can be further classified into 4 categories: nasal, nasal-type, aggressive NK/T-L/leukemia, and blastoid NK cell lymphoma. Extranodal NK/T-L has a greater prevalence in East Asian and Latin American countries than in other parts of the world. The occurence rate in Asia is within 3.3-8\%, with the rate increasing in the order of Japan, Taiwan, Hong Kong, and Korea (3). The origin of the tumor was the nose and paranasal area in more than $80 \%$ of the patients. Extra- 


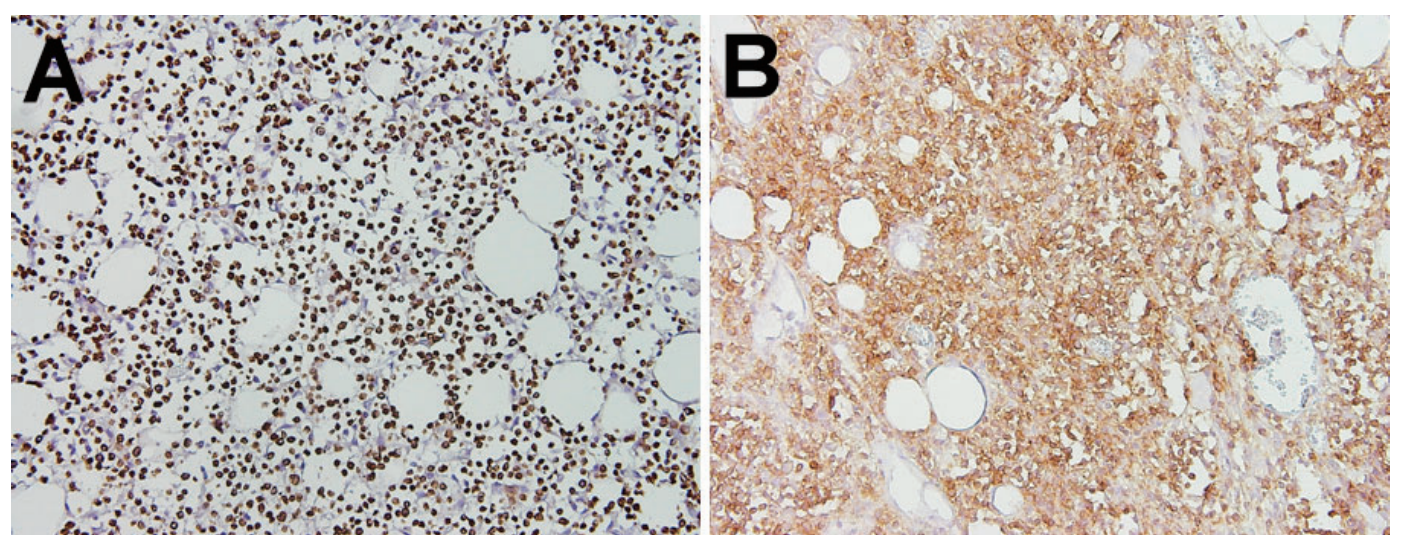

Figure 3. Immunohistochemical characteristics of tumor cells. A: positive staining for CD3; B: positive staining for CD56.

nodal NK/T-L rarely originates in the lung, GI tract, kidney, pancreas, testis, or brain (3).

Lymphomas in the GI tract are difficult to diagnose early due to their nonsepcific symptoms (7). Gastric and colonic lymphomas can be diagnosed via conventional EGD and colonoscopy (1). However, NK/T-L derived from the small intestine, which has a low incidence, is mostly diagnosed incidentally or when the patient presents with acute abdomen. Vigilance against "hints" of small bowel tumors and proper diagnostic tools are the key to prevent the complications, including perforation, massive bleeding, ileus, and to treat the disease when its spread is limited and it is easy to cure.

CA-125 is a glycoprotein found in tissues derived from coelomic epithelia. In the female population, it is a commonly used tumor marker for epithelial ovarian cancer. However, elevated serum CA-125 level has also been reported in nongynecologic tumors and nonmalignant diseases involving the peritoneum, pleura, and pericardium. Zeillemaker et al reported that inflammatory cytokines significantly stimulated the secretion of CA-125 by cultured monolayers of non-activated human mesothelial cells (8). On the basis of the above-mentioned characteristics, elevated levels of CA-125 are indicative of inflammatory, infectious, or neoplastic processes and provide a clue for further investigations. Some reports in the English literature have indicated that serum levels of CA-125 are elevated in cases of NHL. CA-125 was found to be a good marker for follow-up evaluation and may have good prognostic value $(9,10)$. The present patient had intestinal perforation, which was compatible with the high serum level of CA-125 and indicated a delayed diagnosis and poor prognosis. Akiyama et al reported that 4 of 5 natural killer-like $\mathrm{T}$ cell lymphomas involving the small intestine were accompanied by intestinal perforation. The mechanism may be related to the cytotoxic phenotype of lymphoma, which caused damage to tissues with cytotoxic enzymes and led to mucosal ulceration (11). Angiocentricity and angioinvasion of NK/T-L leading to ischemic change may further destroy the bowel wall and result in perforation (12). Therefore, surgical intervention is necessary to prevent perforation in intestinal NK/T-L. We propose that peritoneal involvement secondary to small intestinal perforation is the main reason for the elevation of serum CA-125 level in the present case. Thus, in cases of NK/T-L, a high level of CA-125 is a probable indicator of small intestine perforation and surgical intervention.

Tuberculosis is an endemic infectious disease in several regions, including Taiwan. Several reports have highlighted the remarkable similarity between abdominal $\mathrm{TB}$, ovarian carcinoma, and carcinomatosis peritonei. An elevated CA125 level has been documented in the majority of patients with TB peritonitis. In the present case, it was difficult to differentiate intestinal lymphoma from TB peritonitis by using information gathered by history taking, physical examination, and laboratory tests. ADA, which is widely distributed in tissues and body fluids is a purine-degrading enzyme. The use of a cut-off level of ascetic fluid level of ADA ( $>39$ IU/L) has been reported in the diagnosis of TB peritonitis, with a sensitivity of $100 \%$ and a specificity of $97.2 \%$ (13). In this case, the value of the ascetic fluid level of ADA (30 IU/L) can be used to rule out TB peritonitis. However, ascitic fluid level of ADA may have limited diagnostic value in patients with other underlying diseases. ADA plays a key role in the proliferation and differentiation of $\mathrm{T}$ lymphocytes. Therefore, the use of this marker in cases of T-cell lymphoma or leukemia may lead to the incorrect diagnosis of TB peritonitis. In contrast, cirrhotic patients may have a low ascitic fluid level of ADA because of the dilution of ascitic fluid. An enteroscopy may offer some information in this situation and may be helpful for patients who have no accessible ascitic fluid.

The role of surgical intervention in GI NHL has been a topic of debate. Cai et al reported 43 patients with colon or rectal lymphoma and concluded that the clinical stage plays a more important role in predicting outcome than does the nature of surgical intervention. Although lymphomas can be generalized or systemic, patients who underwent surgery as the first treatment were more likely to be rendered disease free on gross surgical examination than those who underwent chemotherapy as the initial treatment modality because the former would have localized disease amenable to surgi- 
cal resection (14). Thus, surgery should be reserved for patients with localized tumor or complications from diseases or treatment. Because of the high risk of perforation, we suggest that surgical resection of the involved area should be performed to prevent further treatment-related complications, such as perforation or bleeding. However, in patients with considerable spread of tumors, it is necessary that resection be performed skillfully and carefully to prevent complications.

In conclusion, primary small intestinal NK/T-L is an extremely rare condition. Serum CA-125 level, which is solely an indicator of perforation, may also help the prognostic evaluation. The ascitic ADA level could help differentiate this disease from TB peritonitis. Small bowel enteroscopy may offer some information when assessment of the ADA level is not possible. Surgical intervention of intestinal lymphoma, which is reserved for patients with localized tumor or complications, should be performed in cases of intestinal NK/T-L because it has a tendency to cause intestinal perforation.

The authors state that they have no Conflict of Interest (COI).

\section{Acknowledgement}

This study was supported by grant funded by the China Medical University Hospital (DMR-96-028).

\section{References}

1. Kakimoto K, Inoue T, Nishikawa T, et al. Primary CD56+ NK/Tcell lymphoma of the rectum accompanied with refractory ulcerative colitis. J Gastroenterol 43: 576-580, 2008.

2. Kim HS, Lee DK, Baik SK, Kwon SO, Cho MY, Ko YH. Primary CD 56+ T/NK cell lymphoma of the colon. J Gastroenterol 37: 939-946, 2002.
3. Suzuki R, Takeuchi K, Ohshima K, Nakamura S. Extranodal NK/ T-cell lymphoma: diagnosis and treatment cues. Hematol Oncol 26: 66-72, 2008.

4. Barutcu O, Erel HE, Saygili E, Yildirim T, Torun D. Abdominopelvic tuberculosis simulating disseminated ovarian carcinoma with elevated CA-125 level: Report of two cases. Abdom Imaging 27: 465-470, 2002.

5. Simsek H, Savas MC, Kadayifci A, Tatar G. Elevated serum CA 125 concentration in patients with tuberculous peritonitis: a case control study. Am J Gastroenterol 92: 1174-1176, 1997.

6. Mas MR, Comert B, Saglamkaya U, et al. CA-125: a new marker for diagnosis and follow-up of patients with tuberculous peritonitis. Dig Liver Dis 32: 595-597, 2000.

7. Kala Z, Válek V, Kysela P, Svoboda T. A shift in the diagnostics of the small intestine tumors. Eur J Radiol 62: 160-165, 2007.

8. Zeillemaker AM, Verbrugh HA, Hoynck van Papendrecht AA, Leguit P. CA 125 secretion by peritoneal mesothelial cells. J Clin Pathol 47: 263-265, 1994.

9. Benboubker L, Valat C, Linassier C, et al. A new serologic index for low-grade non-Hodgkin's lymphoma based on initial CA125 and LDH serum levels. Ann Oncol 11: 1485-1491, 2000.

10. Bairey O, Blickstein D, Stark P, et al. Serum CA 125 as a prognostic factor in non-Hodgkin's lymphoma. Leuk Lymphoma 44: 1733-1738, 2003.

11. Akiyama T, Okino T, Konishi H, et al. CD8+, CD56+ (Natural killer-like) T-cell lymphoma involving the small intestine with no evidence of enteropathy: Clinicopathology and molecular study of five Japanese patients. Pathol Int 58: 626-634, 2008.

12. Hsiao CH, Lee WI, Chang SL, Su IJ. Angiocentric T-cell lymphoma of the insteine: a distinct etiology of ischemic bowel disease. Gastroenterology 110: 985-990, 1996.

13. Riquelme A, Calvo $M$, Salech $F$, et al. Value of adenosine deaminase (ADA) in ascitic fluid for the diagnosis of tuberculous peritonitis. J Clin Gastroenterol 40: 705-710, 2006.

14. Cai S, Cannizzo F Jr, Bullard Dunn KM, Gibbs JF, Czuczman M, Rajput A. The role of surgical intervention in non-Hodgkin's lymphoma of the colon and rectum. Am J Surg 153: 409-412, 2007.

(C) 2011 The Japanese Society of Internal Medicine http://www.naika.or.jp/imindex.html 